\title{
Adaptive Droop Control of a Multibus DC Microgrid Based on Consensus Algorithm
}

\author{
Mingche Li $\mathbb{D}$, Jiangwei Fan $(\mathbb{D}$, and Lihui Qiao \\ School of New Energy and Power Engineering, Lanzhou Jiaotong University, Lanzhou 730070, China \\ Correspondence should be addressed to Mingche Li; xiaowish913@126.com
}

Received 27 October 2020; Revised 11 December 2020; Accepted 22 December 2020; Published 18 January 2021

Academic Editor: Licheng Wang

Copyright (c) 2021 Mingche Li et al. This is an open access article distributed under the Creative Commons Attribution License, which permits unrestricted use, distribution, and reproduction in any medium, provided the original work is properly cited.

\begin{abstract}
The main control objective of a DC microgrid with a multibus structure is to stabilize the bus voltage and maintain the power balance of the whole system. An adaptive droop control strategy for multibus DC microgrid based on consensus algorithm is proposed. It is based on platform multiagent system, which is realized by network protocol. Under the condition of a weak communication network, the bus at all levels can realize regional power autonomy through packet consensus protocol. A hybrid simulation platform composed of Jade, MacSimJX, and Simulink is built to verify the effectiveness of the control strategy.
\end{abstract}

\section{Introduction}

Nowadays, microgrid is one of the most innovative fields in the power industry. Future microgrid can be used as an energy balance unit of distribution network and as independent power grid to supply community. DC microgrid has the advantages of less conversion times and simple control structure at the same time. It is not necessary to track the phase and frequency of voltage, and the eddy current loss and reactive power compensation in transmission process are not required, which is a more ideal solution for a microgrid system based on new energy generation.

DC bus voltage is the only indicator reflecting the power balance in DC microgrid [1]. Therefore, the important goal of DC microgrid control is to balance the input and output power of the system through the coordinated control of the whole system, thus maintaining the stability of DC bus voltage. The energy coordinated control method based on DC bus signal proposed in reference [2] can only achieve basic power and energy distribution. The DC Bus Signal (DBS) control method cannot achieve ideal power distribution effect because the bus voltage varies with the operating point, the line impedance is unknown, or the distribution is inhomogeneous [3]. However, the point-to-multipoint centralized communication control has strict requirements on the real time performance of the communication network, and its structure is not suitable for the DC microgrid system with microsources and scattered loads. Therefore, the distributed multiagent (MA) consensus and coordination control based on sparse communication network and point-topoint has attracted more and more attention [4]. In 2004, olfati saber first systematically proposed the theoretical framework of a multiagent network consensus problem and gave the basic form of consensus control protocol [5]. Then, Ren and Beard [5] studied the consensus problem of directed weighted networks with fixed topology and switched topology and pointed out that when the system topology contains directed spanning tree, the system can achieve consistency. Compared with literature [6], this conclusion is less conservative. Then, a lot of research and analysis show that the performance of the consensus algorithm is closely related to the connectivity of the network topology. The authors of [4, 7-10] studied the correlation between the connectivity of the communication network and the control gain and the algorithm boundary weight in the microgrid. Among the studies, literature [4] analyzed the limitations of traditional noncommunication control, proposed an adaptive droop control strategy based on discrete consensus, and realized high-precision load distribution and voltage regulation by taking the average voltage difference of the whole network as 
the consensus optimization goal. The authors of $[7,8,11]$ studied the application of consensus algorithm in DC microgrid clusters connected by shared common connection points (PCC), including the implementation of consensus algorithm in the average output current of each microgrid unit and the acquisition of core bus voltage. Literature [9] obtained the global information of the total injected power of nodes and the total number of control terminals participating in optimization, calculated the power and voltage reference values of the local converter station satisfying the specific objective function, and realized the global autonomous decentralized control.

The abovementioned research on DC microgrid consensus is based on a single bus structure, and its optimal control objective is unique. For the regional DC microgrid power supply system in island mode, the double-layer bus structure carries out the high-voltage and low-voltage hierarchical design for the single bus structure, which improves the security and compatibility of low-voltage equipment power supply [12], which is more practical than the single bus structure. Reference [13] proposed a coordinated control strategy for the dual bus DC microgrid. According to the voltage signals of the two buses, the coordinated control strategy and energy management scheme for each microsource and energy converter between the two buses were formulated. However, due to its dependence on the bus voltage signal to judge the working state of the system, high-precision power distribution could not be achieved.

$\mathrm{Yu}$ et al. first studied the group consensus problem of distributed multiagent [14] and obtained some conditions and criteria to ensure the system to achieve group consistency. Through comparative experiments, it was shown that the information interaction among subsystems in the multiagent system can accelerate the convergence speed of the consensus of each subsystem. At the same time, reference [15] studied the grouping consensus problem of multiagent based on discrete time. This is of positive significance to the research on the consensus control of DC microgrid with multibus structure. On this basis, a group consensus control strategy based on multibus DC microgrid is proposed in this paper. Compared with the traditional single bus structure consensus control strategy, it enhances the system compatibility and power allocation optimization ability.

\section{Hierarchical Control of a Multibus DC Microgrid}

2.1. The Problems in the Droop Control without Line Resistance. The Thevenin equivalent model of the doublebus six-node DC microgrid is shown in Figure 1. The traditional control equation of resistive droop is as follows [4]:

$$
U_{\mathrm{oi}}=U_{\mathrm{ref}}-R_{\mathrm{di}} I_{\mathrm{oi}}, \quad i=1,2, \ldots, n_{\mathrm{ode}},
$$

where $U_{\mathrm{oi}}$ is the output voltage of the converter; $U_{\text {ref }}$ is the reference value of the output voltage; $I_{\mathrm{oi}}$ is the output current; $R_{\mathrm{di}}$ is the control coefficient of resistance droop; and $n_{\text {ode }}$ is the total number of nodes. The intermediate energy converter is considered as an ideal energy transmission unit, and it can be seen from equation (1) that to achieve regional power autonomy of the multibus, the output power of the converter should be distributed proportionate to its rated power, and the output voltage and current of each node should meet the following relation:

$$
\frac{I_{\mathrm{oi}}}{I_{\mathrm{oj}}}=\frac{R_{\text {linej }}+R_{\mathrm{dj}}}{R_{\text {linei }}+R_{\mathrm{di}}}=n_{i-j}, \quad i \neq j,
$$

where $n_{i-j}$ is the rated power ratio of the converter $i$ and $j$; $R_{\text {linei }}, R_{\text {linej }}$ is the $i$ and $j$ equivalent line impedance between the converter, corresponding port, and PCC point; Figure 1 shows the equivalent line impedance between high- and lowvoltage buses. It can be seen that when the impedance of the line is complex, the fixed resistive droop control coefficient cannot always meet equation (2), and when the output power of the microsource increases, the output voltage may exceed the limit of $\pm 5 \%$ bus voltage variation.

2.2. Hierarchical Control of Multibus Coordination. The traditional resistive droop control has inherent contradiction between voltage regulation and load proportion distribution, so it is necessary to adjust the control parameters twice, that is, hierarchical control of a DC microgrid.

In this paper, the coordinated layered control of multibus is divided into two layers, namely, equipment level control and system level control, as shown in Figure 2, where, $i_{\mathrm{hi}}, i_{\mathrm{li}}$ and $v_{\mathrm{DChi}}, v_{\mathrm{DCli}}$ are the sampling values of the current and voltage at the terminals of the high- and lowvoltage bus converters. $\Delta U_{\mathrm{hi}}$ and $\Delta U_{\mathrm{li}}$ are the difference between the output voltage state variable of the converter corresponding to the high- and low-voltage bus and the average value of the subnet. In order to avoid the circulating current between nodes in the network and the microsource can adapt to the load power output, the main goal of secondary regulation is to monitor the output voltage and current of each microsource in real time and adjust its output according to the load capacity of the microsource; at the same time, the high-voltage and low-voltage DC buses are each other's hot standby microsources, and they can adjust the power of each other under the premise of surplus capacity making timely supplement.

When adjusting the voltage, the microsource in the network should be adjusted to the average voltage of the high- and low-voltage subnetworks synchronously, as shown in the following equation:

$$
\Delta U=U_{\mathrm{ref}}-\frac{1}{n} \sum_{i=1}^{n_{s}} U_{\mathrm{oi}}
$$

where $n_{s}$ is the total number of nodes corresponding to the subnet.

On this basis, the resistive droop coefficient is changed by the load capacity of each microsource, and its output current is distributed proportionally.

When the number of system nodes increases, it becomes difficult to calculate the target control parameters of quadratic coordination. Compared with centralized control, 




FIgURE 1: Equivalent model of a DC microgrid with two buses.

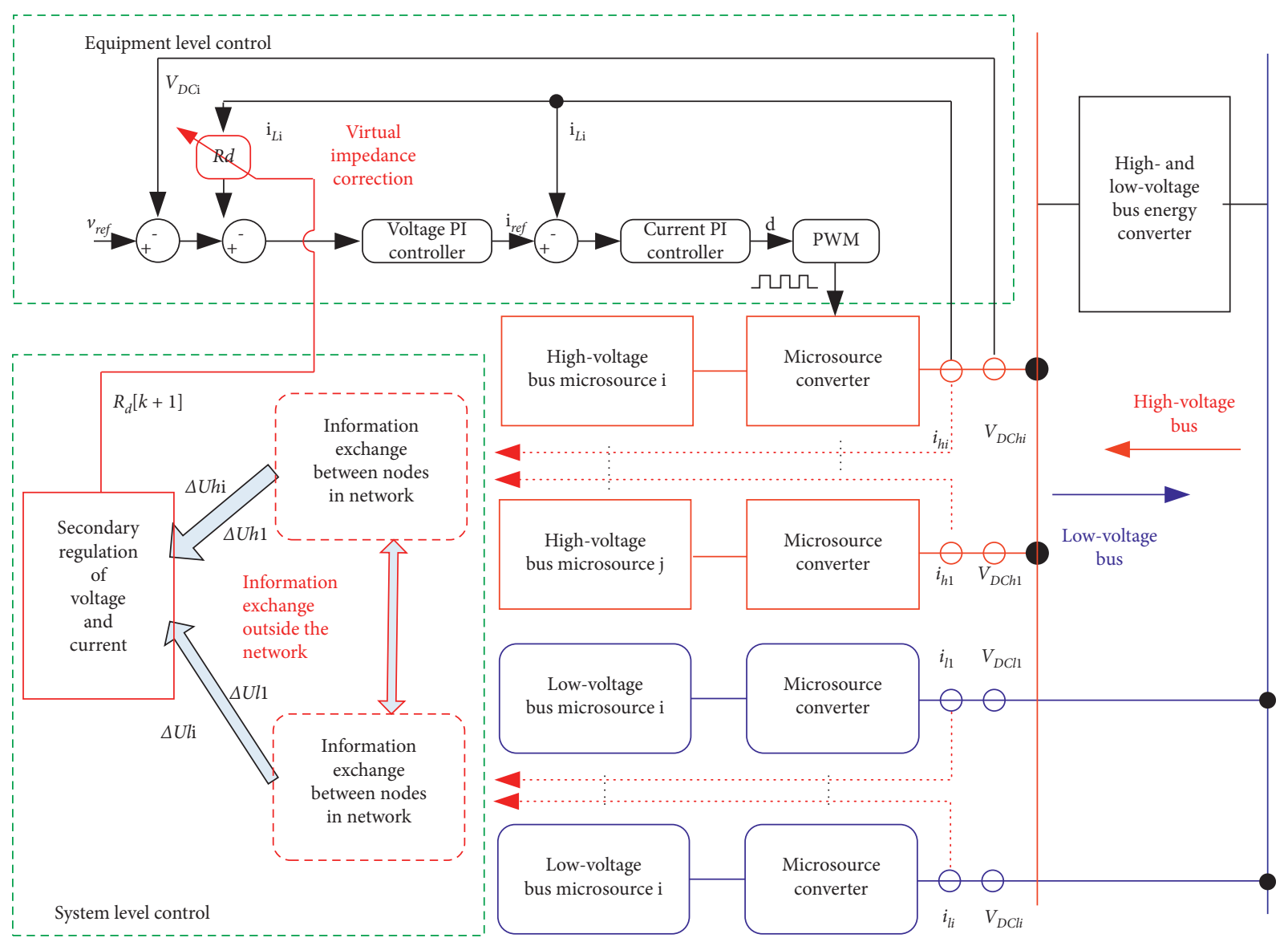

FIgURE 2: Hierarchical control structure of a DC microgrid with two buses. 
distributed control can share the computing pressure of a multinode control system. Therefore, the discrete grouping consensus algorithm is applied in this paper to synchronously calculate the voltage and current control quantities required by high- and low-voltage DC busbars, and the correction quantity of the resistance droop coefficient satisfying each microsource is obtained through the information interaction within and between networks.

\section{Discrete Group Consensus Algorithm}

In the multiagent coordination control, in order to ensure that the target tasks can be distributed and coordinated, the state of all agents needs to be consistent at all times. However, under the influence of environment, state, and even time, the consistent state value of the system will change accordingly. In addition, when multiple different tasks are coordinated by multiple agents, different consistent results will appear, which is the problem of multiagent grouping consensus algorithm. In reference [14], a firstorder linear multiagent group consensus protocol is formulated based on the assumption of degree balance. However, due to the strict assumption that the communication equivalent effect is 0 in internetwork communication, it is not practical. Reference [15] weakens the assumption and no longer requires the communication equivalent effect to be 0 , but it needs new node adjacency weights and assumptions, which still has great limitations. Literature [16], on the basis of its research, formulates a continuous system grouping consensus protocol that is not limited by the abovementioned assumptions, but does not consider the discrete case. Due to the inherent discrete characteristics of data transmission in agent communication and the asynchronous communication mechanism of the Java Agent development framework (JADE), the following discrete packet consensus protocol is considered to be applied in the implementation of the system.

In the first-order discrete multiagent bipartite network with $n+m$ agents, $n$ agents and $m$ agents belong to two different consensus convergence values, and their corresponding nodes belong to two independent system subgraphs $G_{1}$ and $G_{2}$, respectively. The system equation is as follows [18]:

$$
\dot{x}_{i}[k+1]=\dot{x}_{i}[k]+u_{i}[k], \quad i=1,2, \ldots, n+m,
$$

where $x_{i}[k]$ and $u_{i}[k]$ are, respectively, the state value and control input of the $i$ th agent at time $k$. The discrete packet control protocol applied is as follows:

$$
u_{i}[k]=\left\{\begin{array}{l}
\alpha\left[\sum_{v_{j} \in N_{1 i}} a_{i j}\left(x_{j}[k]-x_{i}[k]\right)+\sum_{v_{j} \in N_{2 i}} a_{i j} x_{j}[k]\right], \quad \forall i, j \in G_{L 1}, \\
\alpha\left[\sum_{v_{j} \in N_{2 i}} a_{i j}\left(x_{j}[k]-x_{i}[k]\right)+\sum_{v_{j} \in N_{1 i}} a_{i j} x_{j}[k]\right], \quad \forall i, j \in G_{L 2},
\end{array}\right.
$$

where $a_{i j}$ is the corresponding element of the system adjacency matrix; $G_{L 1}=\{1,2, \ldots, n\}$ and $G_{L 2}=\{n+1, n+2, \ldots, n+m\}$ are the node sequence sets contained in the submatrix of the system Laplacian matrix, which comes from the 2 subsystems described in the division diagram [17], respectively, and the corresponding node set is $N_{1 i}$ and $N_{2 i}$, respectively. $\alpha$ is the system control gain; $\sum_{v_{j} \in N_{\theta i}} a_{i j}\left(x_{j}[k]-x_{i}[k]\right)(\theta=1,2)$ represents the interaction between nodes in the subsystem; $\sum_{v_{j} \in N_{\theta i}} a_{i j} x_{j}[k](\theta=1,2)$ represents the combined influence from other grouped nodes.

For the multiagent network system equation (4), applying the packet control protocol equation (5), the following equation can be obtained:

$$
\begin{gathered}
x[k+1]=\left[\begin{array}{ccc}
I_{n}-\alpha L_{1} & \alpha \boldsymbol{\Omega}_{1} \\
\alpha \boldsymbol{\Omega}_{2} & I_{m}-\alpha L_{2}
\end{array}\right] x[k], \\
\boldsymbol{\Omega}_{1}=\left[\begin{array}{cccc}
a_{1(n+1)} & a_{1(n+2)} & \cdots & a_{1(n+m)} \\
a_{2(n+1)} & a_{2(n+2)} & \cdots & a_{2(n+m)} \\
\vdots & \vdots & & \vdots \\
a_{n(n+1)} & a_{n(n+2)} & \cdots & a_{n(n+m)}
\end{array}\right], \\
\boldsymbol{\Omega}_{2}=\left[\begin{array}{cccc}
a_{(n+1) 1} & a_{(n+1) 2} & \cdots & a_{(n+1) n} \\
a_{(n+2) 1} & a_{(n+2) 2} & \cdots & a_{(n+2) n} \\
\vdots & \vdots & & \vdots \\
a_{(n+m) 1} & a_{(n+m) 2} & \cdots & a_{(n+m) n}
\end{array}\right] .
\end{gathered}
$$


In equation (6), $L_{1}$ and $L_{2}$ are Laplacian matrices corresponding to subgraphs $G_{1}$ and $G_{2}$ and $I_{n}$ and $I_{m}$ are the corresponding dimensional identity matrices. The same assumptions as in reference [19] are adopted:

Assumption 1: for all nodes $i$ in subgraph $G_{1}$, $\sum_{j=n+1}^{n+m} a_{i j}=0$ is satisfied; for all nodes $j$ in subgraph $G_{2}$, $\sum_{j=1}^{n} a_{i j}=0$ is satisfied

Assumption 2: both system subgraphs $G_{1}$ and $G_{2}$ have directed spanning trees corresponding to them

In the actual control system, the convergence performance of the consensus algorithm is an important performance index of the control strategy. In the single-objective consensus discrete control system, the value of the boundary weight $\varepsilon$ is determined by the maximum and submaximum eigenvalues of the Laplacian matrix $L_{s}$ of the system, which is a necessary condition for the fast convergence of the consensus algorithm, as shown in the following equation:

$$
\varepsilon=\frac{2}{\lambda_{1}\left(L_{s}\right)+\lambda_{n-1}\left(L_{s}\right)} .
$$

For the discrete grouping consensus algorithm shown in equation (5), the corresponding control gain $\alpha$ determines whether the system can achieve consistency, as well as fast. It can be obtained by solving the Linear Matrix Inequality (LMI) of the error system based on the topological structure of the system, as shown in the following equations [20]:

$$
\begin{gathered}
{\left[\begin{array}{cc}
\mathbf{P} & \left(I_{n+m-2}+\alpha \mathbf{F}\right)^{T} \\
I_{n+m-2}+\alpha \mathbf{F} & \mathbf{P}^{-1}
\end{array}\right]>0_{2(n+m-2) \times(n+m-2)},} \\
\mathbf{F}=\left[\begin{array}{cc}
-L_{1} & \boldsymbol{\Omega}_{1} \\
\boldsymbol{\Omega}_{2} & -L_{2}
\end{array}\right]
\end{gathered}
$$

where $\mathbf{P}$ is a positive definite matrix and has $\mathbf{P} \in \mathbb{R}^{(n+m-2) \times(n+m-2)}$.

\section{Adaptive Droop Control Based on Group Consensus Algorithm}

4.1. The Overall Architecture of the Control Strategy. In this paper, each unit in the DC microgrid is regarded as an independent agent. Meanwhile, each subsystem can utilize the autonomy and inspiration of the MA system itself to adapt to the control requirements of the decentralized and complex DC microgrid.

During the execution of the control strategy, each agent monitors the output voltage and current of its power unit in real time and collects the information needed by the control. The high-voltage and low-voltage bus agents interact with each other in their own network and obtain the secondary control commands of voltage and current required by each agent through the group consensus algorithm. At the same time, the information exchange between bus boundary nodes can optimize the secondary control performance of the whole system and initiate energy exchange proposal when the power of high- and low-voltage buses is unbalanced. In addition, the system still has the consistent and universal "plug and play" feature, which makes adaptive adjustment when the system topology changes. The equipment level control is responsible for the autonomous regulation of voltage and current, which is completed by the PI controller, as shown in the following equation:

$$
\left\{\begin{aligned}
d & =\left(\frac{K_{\mathrm{ic}}}{s}+K_{\mathrm{pc}}\right)\left(I_{\mathrm{ref}}-I_{i}\right), \\
I_{\mathrm{ref}} & =\left(\frac{K_{\mathrm{iv}}}{s}+K_{\mathrm{pv}}\right)\left(V_{\mathrm{ref}}-V_{i}-I_{i} R_{d}\right),
\end{aligned}\right.
$$

where $s$ is the Laplacian operator; $d$ is the PWM duty ratio; $I_{\text {ref }}$ is the reference value of current; $V_{\text {ref }}$ is the voltage reference value; $K_{\mathrm{ic}}$ and $K_{\mathrm{pc}}$ are current PI controller parameters; $K_{\mathrm{iv}}$ and $K_{\mathrm{pv}}$ are voltage PI controller parameters; and $R_{d}$ is the control coefficient of resistive droop.

Resistive droop control can lead to better current distribution and system damping, but fixed resistive droop coefficient $R_{d}$ cannot bring accurate current distribution. Therefore, the group consensus algorithm is introduced into the system level control to adaptively adjust the resistive droop coefficient by obtaining the average current of the whole network and the average voltage difference of the buses at all levels, so as to realize high-precision load distribution and voltage regulation of the whole network.

4.2. Consensus Iteration of Network Voltage Restoration. The control of resistive droop will inevitably bring about the drop of output voltage of the microsource. Therefore, the adaptive algorithm should first consider the recovery of high- and low-voltage bus voltages. This requires the voltage data of each agent in each subnet to be acquired and processed separately by the group consensus algorithm. The local pressure difference can be obtained from the following equation:

$$
\Delta U_{i}=U_{\mathrm{ref}}-U_{\mathrm{oi}}
$$

Among them, $\Delta U_{i}$ is the local voltage difference of the microsource and $U_{\mathrm{oi}}$ is the output voltage of the microsource.

The local pressure difference is used as the initial value in the agent and the local pressure difference state variables of 
high- and low-voltage buses are updated by the following equation:

$$
\left\{\begin{array}{l}
\Delta U_{\mathrm{hi}}[k]=\alpha\left[\sum_{v_{j} \in N_{\mathrm{hi}}} a_{i j}\left(\Delta U_{\mathrm{hj}}[k]-\Delta U_{\mathrm{hi}}[k]\right)+\sum_{v_{j} \in N_{l i}} a_{i j} \Delta U_{\mathrm{hj}}[k]\right], \quad \forall i \in L_{h}, \\
\Delta U_{\mathrm{li}}[k]=\alpha\left[\sum_{v_{j} \in N_{\mathrm{li}}} a_{i j}\left(\Delta U_{\mathrm{lj}}[k]-\Delta U_{\mathrm{li}}[k]\right)+\sum_{v_{j} \in N_{h i}} a_{\mathrm{ij}} \Delta U_{\mathrm{lj}}[k]\right], \quad \forall i \in L_{l} .
\end{array}\right.
$$

After several iterations, the local pressure difference state variables of each agent will converge to the average local pressure difference $\Delta U_{h}[\infty]$ and $\Delta U_{l}[\infty]$ of the high- and low-voltage bus, respectively.

4.3. Correction of the Droop Coefficient. Because the rated power of the microsource is not the same and its ability to provide external current is also quite different under different working conditions, after obtaining the average pressure difference of each subnet, the output of each microsource should be adjusted according to its load capacity. Equation (14) is used to calculate the per-unit value of each microsource:

$$
I_{i}^{*}=\frac{I_{i}}{I_{i N}},
$$

where $I_{i N}$ is the current rating of the $i$ th microsource. Similar to voltage consensus iterations, information is exchanged through neighbor nodes. According to equation (15), the current control reference values of high- and lowvoltage bus $I_{h_{-} \text {ref }}^{*}$ and $I_{l_{-} \text {ref }}^{*}$ are calculated:

$$
\left\{\begin{array}{l}
I_{h_{\text {_ref }}}^{*}[k]=\alpha\left[\sum_{v_{j} \in N_{\mathrm{hi}}} a_{i j}\left(I_{\mathrm{hj}}^{*}[k]-I_{\mathrm{hi}}^{*}[k]\right)+\sum_{v_{j} \in N_{\mathrm{li}}} a_{i j} I_{\mathrm{hj}}^{*}[k]\right], \quad \forall i \in L_{h}, \\
I_{l_{-} \text {ref }}^{*}[k]=\alpha\left[\sum_{v_{j} \in N_{\mathrm{li}}} a_{i j}\left(I_{\mathrm{lj}}^{*}[k]-I_{\mathrm{li}}^{*}[k]\right)+\sum_{v_{j} \in N_{\mathrm{hi}}} a_{i j} I_{\mathrm{lj}}^{*}[k]\right], \quad \forall i \in L_{l} .
\end{array}\right.
$$

Also, the resistive droop coefficient $R_{d}$ of the corresponding microsource is updated accordingly:

$$
R_{d}[k+1]=\frac{U_{N}-U_{\mathrm{oi}}[k]}{I_{i}^{*}[k] I_{i N} U_{N}}
$$

where $R_{d}[k+1]$ is the coefficient of resistive droop at the $k+1$ th calculation; $U_{\text {oi }}[k]$ and $I_{i}^{*}[k]$ are the actual output voltage and output current per-unit value of the corresponding high-low voltage bus microsource during the $k$ th calculation. $I_{i N}$ is the output current rating of the microsource. $U_{N}$ is the bus voltage rating.

\section{Simulation and Analysis}

In this paper, a hybrid simulation platform based on JADE, MacSimJX, and Simulink is established to verify the effectiveness of the proposed control strategy. The structure of the microgrid is shown in Figure 3. JADE can build a real
MA environment in the local area network. It uses the standard agent communication language ACL (Agent Communication Language) to realize the interaction of the contract network between agents [17]. The five microsource agents are located in two computers in the local area network, and they are interconnected through the TCP/IP protocol of multiple virtual machine terminals. An island DC microgrid model containing 3 high-voltage microsources MGH1-MGH3, 2 low-voltage microsources MGL1 and MGL2, and 3 constant power loads is built in Simulink, and it communicates with JADE through the MacSimJX interface. Each microsource works in the maximum power point tracking mode, and the high- and low-voltage bus voltages are set to $380 \mathrm{~V}$ and $48 \mathrm{~V}$, respectively. The system parameters are shown in Table 1.

5.1. Performance Analysis of Agent Group Consensus Algorithm. The communication topology of each agent in Figure 3 is shown in Figure 4. Assuming that the 




FIgURe 3: Structure of a DC microgrid with two buses.

TABLE 1: Parameters of a DC microgrid.

\begin{tabular}{lccc}
\hline Microsource & Line impedance/ $\Omega$ & Output voltage/ & Output current/A \\
\hline MGH1 & 0.2 & 383 & 8.55 \\
MGH2 & 0.3 & 379 & 8.43 \\
MGH3 & 0.4 & 375 & 7.91 \\
MGL1 & 0.2 & 50 & 20.35 \\
MGL2 & 0.5 & 46 & 20.06 \\
\hline
\end{tabular}

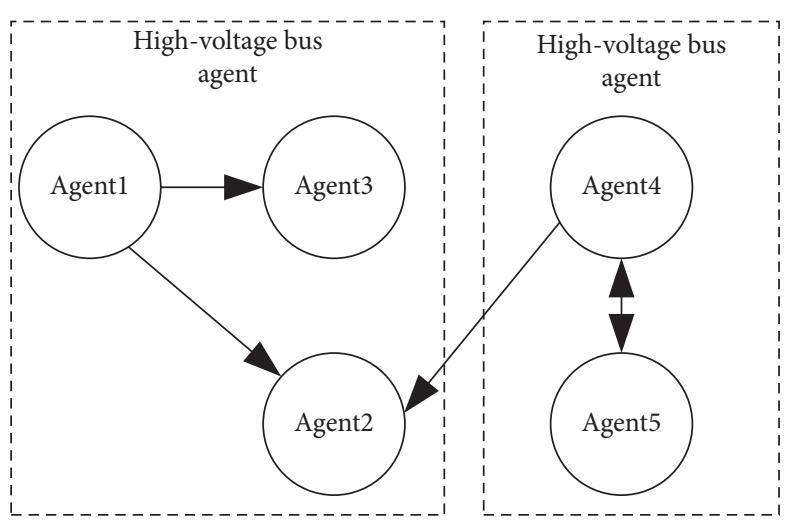

Figure 4: Communication topology structure of agents. 




(a)



(b)

Figure 5: Performance of group consensus algorithm with different control gains. (a) $\alpha=0.3917$. (b) $\alpha=0.6917$.

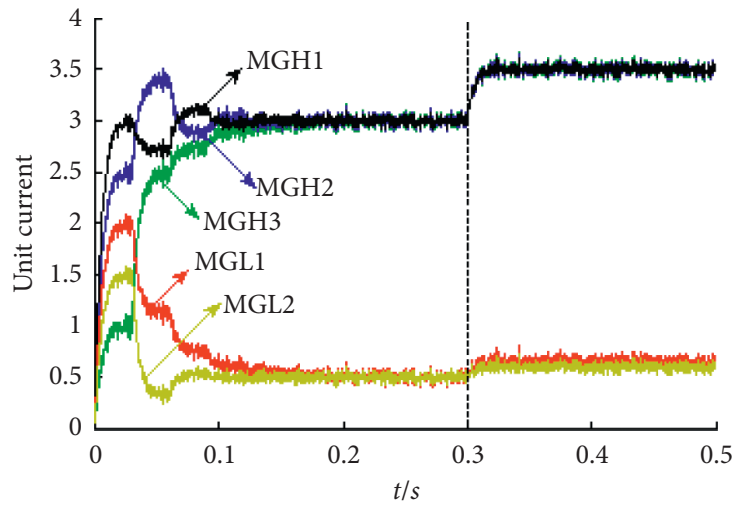

Figure 6: Output currents of microsources with high load disturbance.

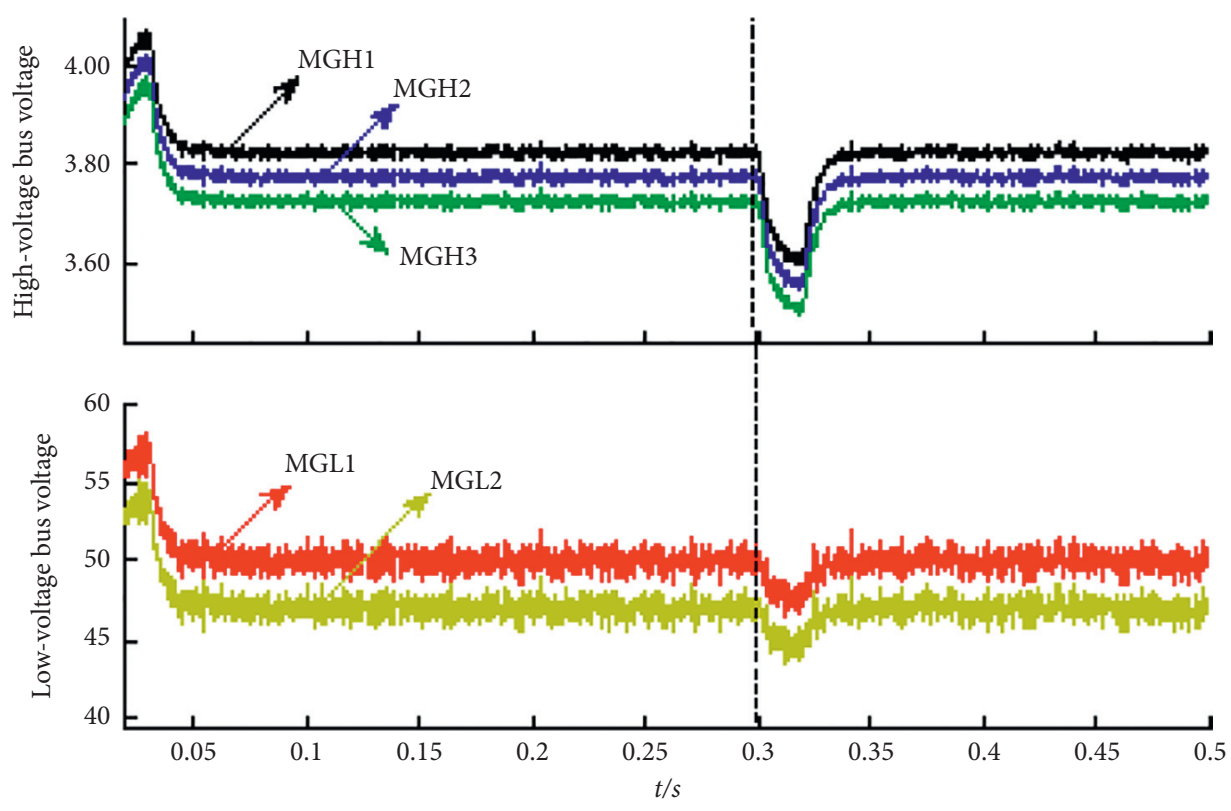

Figure 7: Output voltages of microsources with high load disturbance. 




FIGURE 8: Output currents of microsources with network communication failure.
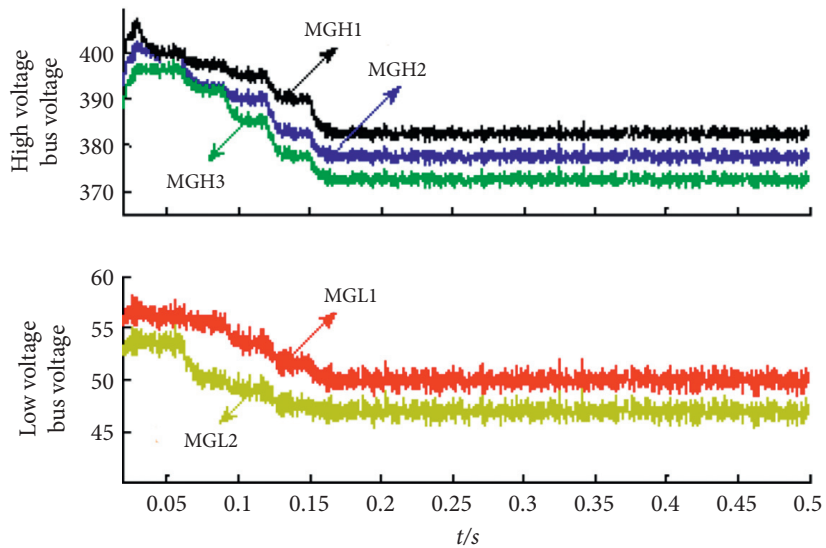

FIGURE 9: Output voltages of microsources with network communication failure.

communication weights are all 1, the Laplacian matrix of each subnet can be obtained as

$$
\begin{aligned}
& \mathbf{L}_{1}=\left[\begin{array}{ccc}
0 & -1 & -1 \\
0 & 2 & 0 \\
0 & 0 & 1
\end{array}\right], \\
& \mathbf{L}_{2}=\left[\begin{array}{cc}
1 & -1 \\
-1 & 1
\end{array}\right] .
\end{aligned}
$$

From equations (6), (7), (9), and (10), combined with the Cone-Complementarity Linearization (CCL) algorithm, the system control gain $\alpha=0.3917$ can be obtained. The convergence characteristics of the consensus algorithm under different control gains are as shown in Figure 5. It can be seen from the simulation results that when $\alpha$ deviating from the optimal solution, the number of iterations of voltage consensus convergence increases significantly, resulting in a decrease in algorithm performance. Among them, the $\alpha$ partial largeness makes the stability of the system worse, and even the consensus convergence cannot be achieved.

5.2. Load Disturbance Analysis. The high-voltage bus of the original system of the DC microgrid has a load of $7 \mathrm{~kW}$, and the low-voltage bus has a load of $1 \mathrm{~kW}$. The redundant power of the system's high-voltage bus is $2.1 \mathrm{~kW}$ during normal operation. The load disturbance analysis is that the highvoltage bus suddenly increases the load of $3 \mathrm{KW}$.

When the sudden load increase is greater than the redundant load capacity of the high-voltage bus, the output current and voltage of the microsource are as shown in Figures 6 and 7, respectively. The high- and low-voltage bus converters work in Boost mode, and the low-voltage bus energy supplements the high-voltage bus. The system reaches power balance in $0.35 \mathrm{~s}$, and the voltage returns to the rated level.

5.3. Communication Failure Analysis. Intranetwork communication failure conditions have been described in literature [19]; here, we consider the case of internetwork communication failures. When the communication link between the two subnets is disconnected, that is, when both, as in equation (6), $\Omega_{1}$ and $\Omega_{2}$ are zero matrixes, the system microsource current and voltage are as shown in Figures 8 and 9, respectively. In the absence of boundary node communication, the proportional distribution of the power of the high- and low-voltage buses of the system under the same control gain takes more time; the bus voltage recovery is also affected by the same effect, and it takes $0.17 \mathrm{~s}$ to recover to the rated level. 


\section{Conclusions}

This paper proposes a distributed collaborative control strategy for an island DC microgrid based on group consensus, and the conclusions are as follows:

(a) The performance of the grouped discrete consensus algorithm under the action of different control gains is quite different, and the CCL algorithm can quickly and accurately determine the gain value suitable for the system.

(b) The application of the group discrete consensus algorithm can make each subsystem achieve their respective consensus convergence faster and has positive significance for the correction control of the secondary current and voltage of the island DC microgrid with a multibus structure.

(c) The system as a whole and each subnet has the "plug and play" feature of distributed power. When the system power changes suddenly, the distributed power in the group communicates through neighbor nodes, the distributed power outside the group communicates through internetwork nodes, and the power needs in the network are proportionally borne by its own ability, which improves the adaptability and reliability of the overall system.

\section{Data Availability}

The data used to support the findings of this study are available from the corresponding author upon request.

\section{Conflicts of Interest}

The authors declare that there are no conflicts of interest.

\section{Acknowledgments}

This work was financially supported by the Youth Program of Lanzhou Jiaotong University and Energy Internet Technology “Tianyou Innovation Team” support plan.

\section{References}

[1] Y. Wang, L. Zhang, H. Li et al., "Hierarchical coordinated control of wind turbine-based DC micro-grid," in Proceedings of the CSEE, vol. 33, no. 4, pp. 16-24, 2013.

[2] X. Chen, M. Shi, H. Sun et al., "Distributed cooperative control and stability analysis of multiple DC electric springs in a DC microgrid," IEEE Transactions on Industrial Electronics, vol. 65, no. 7, 11 pages, 2018.

[3] X. Li, C. Wang, L. Guo et al., "Key technologies of DC microgrids: an overview," Proceedings of the CSEE, vol. 36, no. 1, pp. 2-17, 2016.

[4] L. Ü Zhenyu, Z. Wu, X. Dou et al., "An adaptive droop control for the islanded DC microgrid based on discrete consensus algorithm," Proceedings of the CSEE, vol. 35, no. 17, pp. 4397-4407, 2015.

[5] W. Ren and R. W. Beard, "Consensus seeking in multiagent systems under dynamically changing interaction topologies,"
IEEE Transactions on Automatic Control, vol. 50, no. 5, pp. 655-661, 2005.

[6] R. Olfati-Saber and R. M. Murray, "Consensus problems in networks of agents with switching topology and time-delays," IEEE Transactions on Automatic Control, vol. 49, no. 9, pp. 1520-1533, 2004.

[7] L. Meng, T. Dragicevic, J. C. Vasquez et al., "Modeling and sensitivity analysis of consensus algorithm based distributed hierarchical control for DC microgrids," IEEE Transactions on Smart Grid, vol. 7, no. 3, pp. 1504-1515, 2015.

[8] L. Meng, T. Dragicevic, J. C. Vasquez et al., "Modeling and sensitivity analysis of consensus algorithm based distributed hierarchical control for DC microgrids," in Applied Power Electronics Conference and Exposition, pp. 342-349, IEEE, Charlotte, NC, USA, March 2015.

[9] M. Han, X. U. Dong, L. Wan et al., "Consensus algorithm based decentralized autonomous control of hybrid multiterminal direct current system," Automation of Electric Power Systems, vol. 40, no. 12, pp. 130-136, 2016.

[10] S. Sahoo, J. C.-H. Peng, A. Devakumar, S. Mishra, and T. Dragicevic, "On detection of false data in cooperative DC microgrids-a discordant element approach," IEEE Transactions on Industrial Electronics, vol. 67, no. 8, pp. 6562-6571, 2020.

[11] R. Zhang, B. Hredzak, and T. Morstyn, "Distributed control with virtual capacitance for the voltage restorations, state of charge balancing, and load allocations of heterogeneous energy storages in a DC datacenter microgrid," IEEE Transactions on Energy Conversion, vol. 34, no. 3, pp. 1296-1308, 2019.

[12] X. Yan, Y. Wang, X. Wang et al., "Parallel control based on dynamic consistency for distributed micro-sources," Electric Power Automation Equipment, vol. 37, no. 4, pp. 17-21, 2017.

[13] C. Ren, Y. Zhao, X. Han et al., "Coordination control of DC microgrid with two DC buses," High Voltage Engineering, vol. 42, no. 7, pp. 2166-2173, 2016.

[14] J. Yu and L. Wang, "Group consensus of multi-agent systems with directed information exchange," International Journal of Systems Science, vol. 43, no. 2, pp. 334-348, 2012.

[15] H. Zhao and J. H. Park, "Group consensus of discrete-time multi-agent systems with fixed and stochastic switching topologies," Nonlinear Dynamics, vol. 77, no. 4, pp. 1297-1307, 2014.

[16] C. Tan, G.-P. Liu, and G.-R. Duan, "Group consensus of networked multi-agent systems with directed topology," IFAC Proceedings Volumes, vol. 44, no. 1, pp. 8878-8883, 2011.

[17] F. Bellifemine, Developing Multi-Agent Systems with JADE, National Defense Industry Press, Beijing, China, 2013.

[18] R. Diestel, Graph theory, Higher Education Press, Beijing, China, 4th edition, 2013.

[19] W. Gu, S. Xue, Y. Wang et al., "Finite-time consensus based distributed cooperative control for DC microgrids," Automation of Electric Power Systems, vol. 40, no. 24, pp. 49-56, 2016.

[20] J. Yu and L. Wang, "Group consensus of multi-agent systems with undirected communication graphs," in Asian Control Conference, pp. 105-110, IEEE, Hong Kong, China, August 2009. 